\title{
ERRATA
}

\section{Towards Mesoscopic Thermodynamics: Small Systems in Higher-Order States}

\author{
ROMAN S. INGARDEN \\ Open Sys. Information Dyn. 1, 75 (1992)
}

p. 80 after formula (2.8): A more consequent physical point of view using (2.8) is presented in the new paper by $R$. S. Ingarden and T. Nakagomi, this issuc.

p. 88 formula (6.6) and the 2 nd line: instead of $+\frac{3 N}{2 n}$ should be $-\frac{3 N}{2 n}$.

p. 90 formula (6.13): instead of $\beta_{2}=\frac{1}{U^{2}}\left(\frac{U^{2}}{D^{2}}-\frac{3 N}{2 n}\right)$ should be $\beta_{2}=\frac{1}{2 U^{2}}\left(\frac{U^{2}}{D^{2}}-\frac{3 N}{2 n}\right)$. 